\title{
Características del proceso de construcción del significado del concepto de variación matemática en estudiantes para profesor de matemáticas
}

\author{
Martha Bonilla Estévez, Universidad Distrital Francisco José de Caldas (Colombia) \\ Jaime Romero Cruz, Universidad Distrital Francisco José de Caldas (Colombia) \\ Deissy Narváez Ortiz, Universidad Distrital Francisco José de Caldas (Colombia) \\ Angel Bohórquez Arenas, Universidad Distrital Francisco José de Caldas (Colombia)
}

Recibido el 10 de Noviembre de 2014; aceptado el 25 de Febrero de 2015

Características del proceso de construcción del significado del concepto de variación matemática en estudiantes para profesor de matemáticas

\section{Resumen}

Este artículo caracteriza el proceso de construcción del significado de variación matemática de un grupo de 24 estudiantes para profesor de matemáticas. El experimento de enseñanza se organizó alrededor de la resolución de dos problemas de optimización utilizando la derivada y la programación lineal como dos modelos matemáticos para la variación. El análisis se centró en cómo emergían los rasgos característicos de una comunidad de aprendices en las sesiones de resolución de problemas de variación. Los resultados indican, en primer lugar, que los estudiantes para profesores construyen el significado de variación matemática vinculado al significado de variable, parámetro, cuantificadores, relaciones funcionales y relaciones de covariación. En segundo lugar, la constitución de una comunidad de práctica se apoyó en la manera en la que los estudiantes para profesor identificaron objetivos comunes constituyendo espacios de intercambio y desarrollando sistemas de simbolización compartidos.

Palabras clave. Formación de profesores; Aprendizaje de la variación; Comunidad de aprendizaje; Resolución de problemas; Variación matemática.

Características o processo de construção do significado de variação matemática em futuros professores de matemática

\section{Resumo}

Este artigo caracteriza o processo de construção do significado de variação matemática de um grupo de 24 futuros professores de matemática. O experimento de ensino organizou-se entorno à resolução de dois problemas de optimização utilizando a derivada e a programação lineal como dois modelos matemáticos para a variação. A análise centrou-se em como emergiam os rasgos característicos de uma comunidade de aprendizes nas sessões de resolução de problemas de variação. Os resultados indicam, em primeiro lugar, que os estudantes para professores constroem o significado

Para citar: Bonilla, M., Romero, J., Narváez, D. \& Bohórquez, A. (2015). Características del proceso de construcción del significado del concepto de variación matemática en estudiantes para profesor de matemáticas. Avances de Investigación en Educación Matemática, 7, 73 - 93.

(C) Sociedad Española de Investigación en Educción Matemática (SEIEM). www.seiem.es 
de variação matemática vinculado ao significado de variável, parâmetro, quantificadores, relações funcionais e relações de covariação. Em segundo lugar, a constituição de uma comunidade de prática apoiou-se na maneira na qual os estudantes para professor identificaram objetivos comuns constituindo espaços de intercâmbio e desenvolvendo sistemas de simbolização partilhados.

Palavras chave. Formação de professores; Aprendizado da variação; Comunidade de aprendizado; Resolução de problemas; Variação matemática.

\section{Characteristics of the building process of mathematic variation concept meaning in prospective teachers of mathematics}

\section{Abstract}

This article characterizes the building process of the meaning of mathematic variation in 24 prospective teachers of mathematics. The teaching experiment was organized around the solution process of two optimization problems using the derivative and linear programming as two mathematical models for variation. The analysis was centered in how the characteristic features of a community of learners emerged in the resolution sessions of the variation problems. The results indicate, in first place, that the students to be teachers build the meaning of mathematic variation linked to the meaning of variable, parameter, quantifiers, functional relations and covariation relations. In second place, the community of practice's creation was based on the way the students to be teachers identified commune objectives constituting spaces of interchange and developing shared symbolization systems.

Key Words. Teacher Education; Learning of variation; learning community; Problem solving; Mathematical variation.

Caractéristiques du processus de construction du signifié de variation mathématique dans les étudiants pour professeur de mathématiques

\section{Résumé}

Cet article caractérise le processus de construction du signifié de variation mathématique d'un groupe de 24 étudiants pour professeur de mathématiques. L'expérience d'enseignement a été organisée autour de la résolution de deux problèmes d'optimisation en utilisant la dérivée et la programmation linéaire comme deux modèles mathématiques pour la variation. L'analysé s'est concentrée sur comment émergeaient les traits caractéristiques d'une communauté d'apprentis dans les séances de résolution de problèmes de variation. Les résultats indiquent, en premier lieu, que les étudiants pour des professeurs construisent le signifié de variation mathématique lié au signifié de variable, de paramètre, des quantificateurs, des relations fonctionnelles et des relations de covariation. En deuxième lieu, la constitution d'une communauté de pratique on l'a appuyée dans la manière dans laquelle les étudiants pour professeur ont identifié des objectifs communs en constituant des espaces d'échange et en développant des systèmes partagés de symbolisation.

Paroles clés. Formation des enseignants; L'apprentissage de la variation; communauté d'apprentissage; Résolution de problèmes; variation mathématique.

\section{Introducción}

El aprendizaje de las matemáticas por parte de los estudiantes para profesor es un foco de interés en la formación de profesores de matemáticas (Sowder, 2007). Una de las funciones de los programas de formación es ayudar a los estudiantes para profesor a desarrollar el conocimiento de matemáticas pertinente para la labor de enseñar en contextos que les permitan desarrollar reflexiones didácticas sobre la propia actividad matemática. Una manera de dar cuenta de estos principios es que los programas de formación puedan desarrollar experiencias formativas que permitan a los estudiantes para profesor relacionar su propio aprendizaje con la labor docente de enseñar matemáticas (Fernandes, Lester, Borralho, \& Vale, 1997). La hipótesis es que los 
estudiantes para profesor puedan construir de manera simultánea conocimiento matemático y modos de pensar y actuar que les permitan abordar tareas profesionales. Esta hipótesis genera cuestiones relativas al diseño de entornos de aprendizaje en los programas de formación como "oportunidades" que permitan a los estudiantes para profesor construir conocimiento matemático y tener la oportunidad de reflexionar sobre el proceso de enseñanza-aprendizaje. En este contexto, las aproximaciones a través de la resolución de problemas proporcionan los ingredientes para implicar a los estudiantes en la actividad matemática que puede ayudarles a profundizar su comprensión de las matemáticas como profesores (Biza, Nardi, \& Zachariades, 2007; Bohórquez, Bonilla \& Romero, 2009; Conner, Wilson, \& Kim, 2011; Santos-Trigo \& Camacho-Machin, 2009). En particular, uno de los ámbitos donde la formación de profesores de educación secundaria y las aproximaciones a través de la resolución de problemas se relacionan es en la enseñanza del Cálculo.

\subsection{La variación como contenido de aprendizaje de los estudiantes para profesores}

El aprendizaje de los diferentes conceptos matemáticos en el dominio del Cálculo ha sido objeto de investigación durante tiempo (Artigue, 1991; Artigue, Batanero, \& Kent, 2007; Dreyfus, 1990; Selden \& Selden, 2001). Sin embargo, adoptar una perspectiva desde la formación de profesores las investigaciones en este ámbito son más escasas (Garcia, Llinares, \& Sánchez-Matamoros, 2011; Sánchez-Matamoros, Fernández, \& Llinares, 2014; Sánchez-Matamoros, García \& Llinares, 2006; Silver, Clark, Ghousseini, Charalambous, \& Sealy, 2007). Por otra parte, desde una perspectiva curricular en formación de profesores de matemáticas de educación secundaria algunas propuestas han empezado a integrar los principios derivados de la resolución de problemas y cuestiones relativas al aprendizaje de los conceptos del Cálculo (LEBEM, 1998).

La variación matemática se entiende como la cuantificación del cambio en diversas clases de situaciones con magnitudes continuas y discretas (Cantoral \& Farfán, 1998; Cantoral, Molina, \& Sánchez, 2005). En particular se enfatiza que cambios en una(s) variables producen cambios simultáneos en las otras y que para solucionar dichas situaciones se requiere identificar la forma de la relación y cuantificarla. Desde estas perspectivas, la variación matemática hace parte de una amplia estructura conformada por distintas formas de variación que modela infinitud de situaciones en la que está presente alguna forma de cambio y su cuantificación. Esto puede traducirse en proposiciones matemáticas que involucran el reconocimiento de variables, parámetros, cuantificadores, relaciones funcionales y relaciones de covariación, y un objetivo: la optimización. En nuestra experimentación en formación de profesores y adoptando una aproximación basada en la resolución de problemas consideramos dos contextos de variación matemática: el modelo de derivada en el que las situaciones se caracterizan por el manejo de magnitudes continuas y un comportamiento que se pretende optimizar y, el de la programación lineal en la que las magnitudes son discretas y se pretende encontrar un valor óptimo entre los casos posibles.

Este artículo presenta las características de la manera en la que un grupo de estudiantes para profesor de matemáticas construyen el significado del concepto de variación en un contexto que enfatiza la resolución de problemas en una comunidad de práctica. 


\section{Marco conceptual}

Desde perspectivas socioculturales del aprendizaje se subraya que se aprende a partir de la participación y negociación de significados en contextos de resolución de problemas (Wenger, 2001). El aprendizaje se entiende como la participación paulatina en una comunidad de práctica (Lave \& Wenger, 1991). En un contexto de formación de profesores en el que se crean oportunidades para la resolución de problemas, las características de la comunidad de práctica vienen determinadas por la manera en la que los estudiantes para profesor se involucran en procesos de negociación de los significados de los elementos matemáticos que constituyen el foco del aprendizaje. Ver el aprendizaje de los estudiantes para profesor desde la perspectiva de la negociación de los significados implica poder discutir diferentes interpretaciones de los elementos matemáticos y de sistemas de símbolos usados como instrumentos para articular la comunicación en la comunidad. De esta manera, la noción de comunidad de aprendices (adaptando el significado de comunidad de práctica en contextos de formación de profesores) enfatiza el proceso social de adoptar objetivos compartidos. Por otra parte, Wenger (2001) mantiene que las personas ganan experiencia de su mundo a través de la negociación de los significados que implica la interacción de dos procesos, la participación y la objetivación (formas de identificar lo que es relevante para comprender las situaciones de resolución de problemas en las que se encuentran). De esta manera, las formas de participación de los estudiantes para profesor durante la resolución de problemas pueden ayudar a generar rasgos de lo que constituye una comunidad de aprendizaje caracterizada a través de:

1) la construcción de dominios de interés común,

2) la participación en actividades conjuntas, discusiones y formas de compartir información, $\mathrm{y}$,

3) el desarrollo de un repertorio compartido de recursos. En el caso particular de situaciones de resolución de problemas de variación en los programas de formación, los recursos útiles son la simbología matemática de variable, función, covariación, razón de cambio y el discurso matemático generado.

Teniendo en cuenta estas referencias previas en un contexto de resolución de problemas planteado con el objetivo de generar procesos de significación del concepto de variación matemática con estudiantes para profesores de educación secundaria, nos planteamos la siguiente pregunta de investigación:

¿Cómo el significado de la variación matemática es construido por un grupo de estudiantes para profesor cuando participan en un contexto de resolución de problemas desde la perspectiva de una comunidad de aprendizaje?

\section{Metodología}

\subsection{Participantes y contexto}

En el experimento de enseñanza participaron 24 estudiantes para profesor del curso de didáctica de la variación. Estos estudiantes habían cursado asignaturas sobre aritmética y su didáctica, álgebra y su didáctica con énfasis en los procesos de generalización e introducción a las relaciones funcionales y su didáctica, la transición aritmética-álgebra y sobre problemas del continuo (LEBEM, 1998). Durante el 
experimento de enseñanza, cursaron simultáneamente la asignatura sobre problemas del movimiento en donde abordaban la noción de derivada y aspectos relativos a la densidad de los números reales, pero aún no habían estudiado programación lineal.

Se tomaron datos de 16 estudiantes organizados en cuatro grupos: G1 (5 estudiantes), G2 (3 estudiantes), G3 (4 estudiantes) y G6 (4 estudiantes) para realizar un análisis detallado de cómo dotaban de significado a la variación matemática. G1 se eligió por la experiencia previa de algunos de sus miembros en ser filmados y grabados en audio y porque su proceso de resolución del problema de la viga (uno de los problemas-proyectos planteados en el experimento de enseñanza) aportaba numerosas características de cómo se estaba construyendo los significados de variación tanto en el nivel de grupo pequeño como durante sus participaciones en las discusiones en gran grupo. G2 se eligió porque fue el único en centrar su trabajo en la resolución del segundo problema, la fábrica, y G3 por la manera en que asumieron el rol de profesor cuando expusieron sus procesos de resolución al gran grupo. G6 fue elegido por su participación en las discusiones en gran grupo que permitió la emergencia de contextos en los que se compartían los significados.

Los datos, provienen de las transcripciones de los audios y videos de las interacciones de los integrantes grupos G1, G2 y G3 en la resolución del problema, así como las interacciones del gran grupo, de los cuadernos de cada uno de los integrantes de estos grupos, de los informes entregados por los grupos G1, G2 y G3.

\subsection{Experimento de enseñanza}

La aproximación a los fenómenos educativos a través de los experimentos de enseñanza (Design-Based Researcher Collective, 2003; Gravemeijer, 2004; Simon, 2000) sigue un ciclo de tres fases (Cobb, Confrey, diSessa, \& Lehrer, 2003; Steffe, Thompson \& von Glasersfeld, 2000): Diseño y planificación, experimentación en el aula y análisis retrospectivo.

El experimento de enseñanza diseñado estuvo formado por 28 sesiones de dos horas cada una agrupadas en tres ciclos. El primer ciclo estaba formado por 16 sesiones centradas en la resolución de los problemas. El segundo formado por 10 sesiones centradas en el análisis didáctico de los problemas de variación. El tercero estaba formado por 2 sesiones centradas en el análisis y reflexión de la resolución de problemas como contexto de aprendizaje. Inicialmente se propusieron dos problemas solicitando a cada grupo elegir uno para su resolución. Finalizado el proceso de resolución debían presentar y discutir sus producciones ante el gran grupo. Los estudiantes para profesor podían utilizar toda clase de recursos digitales o analógicos para sus aproximaciones a la resolución. De los cuatro grupos sobre los que hemos centrado el análisis, G1, G3 y G6 eligieron el problema de las vigas y G2 el problema de la fábrica. Este hecho permitió que se generara un contexto de intercambio de significados durante las discusiones colectivas en gran grupo que determinó el proceso de significación de la variación matemática abarcando contextos continuos y discretos.

\section{Los dos problemas propuestos}

Los problemas permiten hacer un abordaje del concepto de variación como cuantificación de la medida del cambio que puede ser expresada por distintos modelos matemáticos, la derivada como una medida de la velocidad de cambio en un contexto continuo y la programación lineal como un contexto para modelar la covariación con magnitudes discretas. Estaban dirigidos a que los estudiantes para profesor generaran 
significado del concepto de variación que involucra el reconocimiento de las variables, parámetros, cuantificadores, relaciones funcionales y relaciones de covariación y un objetivo: la optimización.

Problema 1. La resistencia de la Viga. La resistencia de una viga de sección rectangular es proporcional al producto de su ancho $a$ por el cuadrado de su altura $h$. Se quiere aserrar un tronco de madera de forma cilíndrica con diámetro $\varphi$ $\emptyset$ dado, una viga de sección rectangular. Pierre asegura que es posible encontrar las dimensiones de la viga de mayor resistencia, calculándolas por medio de pequeñas alteraciones a una de las dimensiones. ¿Estás de acuerdo con esta afirmación?
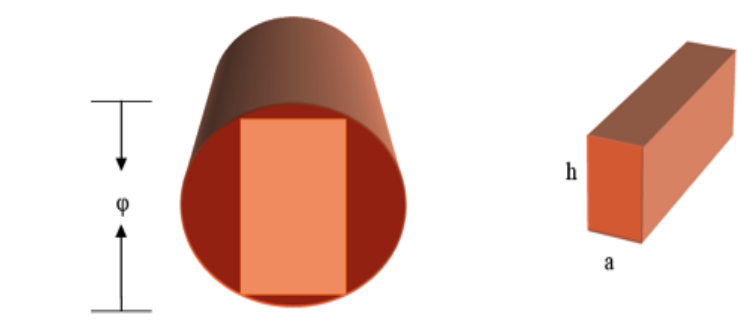

Figura 1. Problema de la viga

Este problema permite abordar el concepto de variación como la cuantificación de la medida del cambio, ya que modela la situación entendiendo la covariación como la razón de cambio entre la resistencia y una de las dimensiones de la sección rectangular. Expresando la relación de las tres variables a, h y $\mathrm{r}$ en términos del parámetro (diámetro del tronco), teniendo en cuenta la relación pitagórica y las restricciones dadas en el problema.

Problema 2. El problema de la fábrica. Una fábrica de partes de avión hace motores y hélices. El costo de producción de los dos productos, no puede superar el millón de dólares. La empresa está entrando en liquidación y necesita ejecutar la mayor cantidad del presupuesto. ¿Cuál es y cómo se adquiere el mayor costo de producción en función de las unidades vendidas de cada una?

Figura 2. Problema de la fábrica

Este problema permite abordar el concepto de variación como la cuantificación de la medida de cambio, en tanto modelo lineal de un costo, expresando la relación de lo que aporta al costo producir una unidad de hélice o una unidad de motor. Para su solución es necesario establecer que las variables (número de motores y número de hélices) cumplen con una relación lineal cuya restricción corresponde al costo de producción, y que esta relación muestra la covariación del aporte de cada una de las dos variables al costo.

\subsection{Análisis}

Los datos de esta investigación son las transcripciones de los audios y videos de las interacciones de los integrantes de G1, G2, G3 y G6 en la resolución del problema, las interacciones del gran grupo, los informes entregados por estos grupos y los cuadernos de resolutor de cada uno de los integrantes. Para describir los procesos de construcción del significado del concepto de variación adaptamos la noción de viñeta 
(Gavilán, García, \& Llinares, 2007) y usamos como descriptores del concepto de variación las variables, parámetros, cuantificadores, relaciones funcionales y relaciones de covariación y optimización, mencionados en el apartado anterior. Para describir el proceso de construcción del significado de variación, en su dimensión social usamos los descriptores de la constitución de una comunidad de práctica (Wenger, 2001). La noción de viñeta permite evidenciar los rasgos característicos del proceso de significación del concepto de variación matemática generado en una comunidad de aprendices. La construcción de las dos viñetas presentadas en los resultados se inicia con la extracción de datos obtenidos en varias sesiones de clase, que permiten mostrar la búsqueda por parte de los estudiantes para profesor de un significado común del concepto de variación en el contexto continuo y la integración de los significados del concepto de variación procedentes de los dos problemas como casos particulares de un significado del concepto de variación.

Con relación a la búsqueda de un significado común de variación los datos se organizaron de manera cronológica, a partir de la primera socialización en gran grupo. Estos datos, dan cuenta de cómo lo presentado por G3 sobre su proceso de resolución y sus consideraciones sobre el concepto de variación son tenidos en cuenta en sesiones siguientes por G1. Respecto a la integración de los significados de variación procedentes de los dos problemas, los datos se organizan teniendo en cuenta momentos claves de interacción en donde los pequeños grupos debieron dar cuenta de su proceso de resolución y sus apreciaciones sobre el significado de variación presentada por otro grupo. Estos datos muestran cómo los grupos construyeron su significado de variación y cómo llegan a ser conscientes de que cada tipo de problema es un caso particular del concepto de variación.

La descripción de la dimensión social en el proceso de construcción del significado de variación usa las características de una comunidad de práctica adaptadas a fin de describir la emergencia y sostenibilidad de una comunidad de aprendices en un contexto de formación de profesores (Wenger, 2001). Estas características son:

- El proceso por el cual los estudiantes para profesor construyeron dominios comunes de interés para hacer emerger significados compartidos sobre los diferentes elementos que configuraron el concepto de variación.

- La manera en la que se implicaron en la realización de actividades, discusiones y el contexto interactivo para compartir información.

- La manera en la que desarrollaron un repertorio de recursos para la resolución de los problemas, tales como el uso de los sistemas de símbolos para representar las relaciones funcionales en el concepto de covariación y la manera en la que el discurso elaborado puede ser entendido como un discurso matemático dirigido a los demás con intención de generar posibilidades de acción en sus compañeros.

\section{Resultados}

La identificación e interpretación de las características del proceso de construcción del significado de variación ha sido organizado en dos apartados. En el primero a través de dos viñetas damos cuenta del proceso de construcción del significado del concepto de variación. En el segundo se presenta la emergencia de los tres aspectos que dan cuenta de la construcción de la comunidad de aprendices. 


\subsection{La constitución del significado de variación}

El proceso de construcción del significado de variación se describe utilizando las interacciones que se dieron para construir significados compartidos de variación en el contexto continuo y para construir un significado más general del concepto de variación. Usamos las interacciones entre los estudiantes para profesor para dar cuenta de la manera en la que se ven los intentos por integrar en un significado de variación general los significados parciales que emergen en los contextos continuo y discreto.

Viñeta 1: Búsqueda de un significado común del concepto de variación en el contexto continuo

Los datos que constituyen esta viñeta proceden de las sesiones séptima y octava. En estas sesiones G1 y G3 presentan los procesos de resolución del problema de la Viga. En esta exposición G1 describe su proceso de solución, caracterizado inicialmente por considerar que la sección de la viga era un cuadrado $(a=h) y$, posteriormente modificando artiméticamente los valores de los lados para determinar cómo afecta esta variación al valor de la resistencia teniendo en cuenta la condición dada en el enunciado del problema.

G3 presenta la utilidad de establecer la relación del diámetro del tronco con las magnitudes ancho y altura de dicha sección rectangular. G3 concluye que esta relación está asociada directamente al teorema de Pitágoras. Al finalizar la exposición, algunos estudiantes les solicitan describir nuevamente cómo relacionan el diámetro $\varnothing$, con el teorema de Pitágoras. En el siguiente fragmento Luis, miembro de G3, indica:

“Entonces por el Teorema de Pitágoras sacamos que ' $h$ ' al cuadrado es igual a la suma de los dos catetos al cuadrado, entonces despejamos y nos quedó que $\varphi$ al cuadrado es igual a la altura al cuadrado más la anchura al cuadrado". [Sesión 7, G3, exposición en gran grupo]. Realiza el siguiente gráfico (Figura 3)

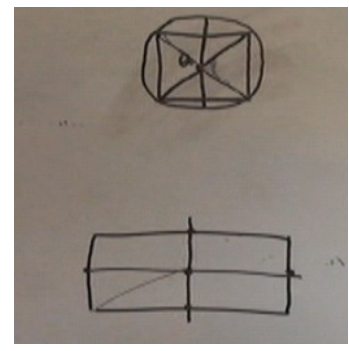

Figura 3. Dibujo realizado por Luis para apoyar su argumento

y asegura:

“... nos quedó que la anchura al cuadrado, que es la que queremos averiguar porque estamos variando la altura, da igual a $\emptyset$ al cuadrado menos el cuadrado $d e$ " $h$ ". Y ahí, ya comenzamos a sacar ... a ver cómo variaba..." [Sesión 7, G3, exposición en gran grupo].

Luis concluye su explicación sobre la utilidad del teorema de Pitágoras afirmando:

"Ya con esta regla, o sea ya aplicándole el teorema de Pitágoras pudimos sacar, digamos como una tabla más organizada, donde tomamos $h$, lo íbamos variando en 0,02 y poníamos en el primer caso, cuando $k$ es igual a uno. Y asi pues, ahí 
averiguábamos el valor de h (Alude al valor del ancho, a, como se muestra en la figura 4). [Sesión 7, G3, exposición en gran grupo].

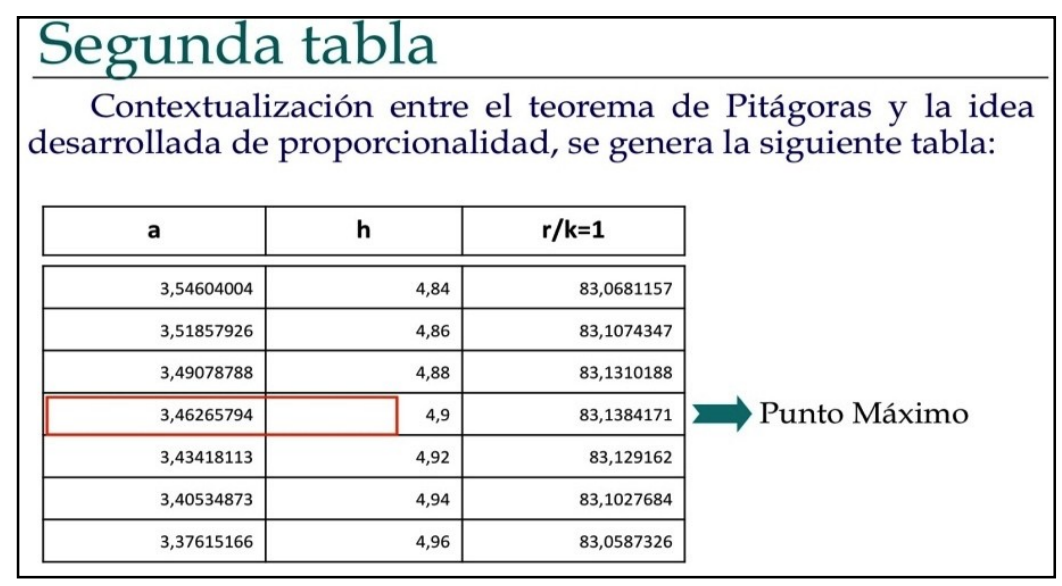

Figura 4. Tabla de datos usada por G3 para apoyar su argumentación

Esta apreciación tiene en cuenta que la sección rectangular está inscrita en el círculo y que pueden expresar dos magnitudes que varían en términos de una sola, identificando las relaciones entre las variables y las restricciones como un elemento del significado de la variación.

En la octava sesión los estudiantes se reúnen en grupos pequeños y los miembros de G1 tratan de describir el procedimiento seguido por G3. Deciden realizar una tabla usando la relación pitagórica de la forma como se presentó en la sesión anterior. Al comienzo observan valores específicos que producen resistencias mayores intentando ver con casos particulares cómo se comporta la función, más adelante utilizan las tablas para determinar qué quiere decir pequeña alteración en los valores de una variable en relación con el comportamiento que estas alteraciones producen en la otra variable.

Julián: ¿cómo generalizan $\emptyset$ [diámetro] ellos? [Alude a lo dicho por G3 en la sesión anterior], es que yo no vi, creo que es por las tablas

Lady: por Pitágoras

Pablo: creeríamos que es las tablas

Miguel: ayúdenme a terminarlo, entonces hagamos uno [una viga] que tenga los lados cuadrados, digo que sea un cuadrado, que tenga los lados iguales y a h le agregamos 0.5 periódico y hallar a. [Sesión 8, G1, trabajando en grupo pequeño].

Este intercambio pone de manifiesto que los integrantes de G1 intentan comprender la manera como sus compañeros de G3 usaron la relación pitagórica, que es diferente y más eficaz que la manera en que ellos la usaron.

Miguel: No, por Pitágoras, como ya tenemos el diámetro, entonces sería, diámetro al cuadrado menos hal cuadrado igual a $\varphi$ al cuadrado.

Julián: usted está hallando de una vez la resistencia, ¿y para generalizar $\varphi$ ?

Lady: ahi generalizar $\varphi$ es que yo reemplacé $\varphi$ por cualquier valor y para todos los valores va a ser... $\varphi$ es un valor independiente.

Pablo: ¿y los valores dependientes cuáles son? 
Miguel: $a y h$.

Lady: dependientes de $\varphi$ y además a dependiente de $h$ [Sesión 8, G1, trabajo en grupo pequeño]

En este intercambio se observa que los estudiantes de G1 encuentran que la relación pitagórica les permite despejar $\mathrm{h}$ en función de a, lo que les posibilitará establecer la resistencia en función de una sola variable. Este procedimiento puede ser entendido como un intento por ponerse de acuerdo sobre el uso de la restricción para expresar la función a optimizar en términos de una sola variable -técnica estándar usada en procesos de cuantificar el cambio-. En esta interacción se revelan las aproximaciones de los estudiantes para comprender algunos de los diferentes aspectos que constituyen una manera de modelizar la variación en contexto continuo (identificar las variables, relaciones entre las variables y restricciones, formas de simbolizar para establecer las relaciones de covariación) entendidas como una medida de la velocidad de cambio - modelo de derivada-).

Viñeta 2: Integrando los significados de variación procedentes de los dos problemas como casos particulares de un significado general de variación

Los datos proceden de las sesiones 10, 15 y 28. En la sesión 28 los grupos presentaban las respuestas a las preguntas del informe-síntesis. Los estudiantes han resuelto el problema de la viga o de la fábrica, han identificado los elementos característicos del concepto de variación procedente del proceso de resolución seguido en cada uno de los problemas -variables de la situación, parámetros, relaciones, restricciones, medida de cambio-. En este informe-síntesis se pretendía que los estudiantes reflexionaran sobre lo aprendido de variación (elementos característicos y formas de representarlos). Parte de la tarea consistió en ver el video en el que David (miembro de G3) exponía como resolvió el problema de la viga. David pone de manifiesto un significado de variación que incluye reconocimiento de las variables, parámetros y cuantificadores; establece la relación funcional y en especial la covariación, explicando que existe una relación entre los cambios que se dan en las resistencias y los cambios en la magnitud de uno de los lados de la sección de la viga y que la relación está cuantificada por una razón. El informe-síntesis incluía responder la pregunta ¿Los elementos que ayudan a constituir el significado de variación pueden identificarse en el proceso de resolución seguido por usted?

Cuando G2 respondía la pregunta, Edna y Adriana (integrantes de G2) presentaron la dependencia entre variables como algo común entre lo expuesto por David y su propio procedimiento, afirmando que el cambio de una(s) variable(s) genera cambios en la otra(s) variable(s), haciendo mención a la presencia de las condiciones:

"Durante el proceso de resolución en los dos ciclos del problema pudimos evidenciar diferentes formas de ver la variación, sin embargo cada ciclo trajo consigo diferentes concepciones de este concepto. En el momento que decidimos modificar el problema observamos que cumplía con muchos de los aspectos del problema de la viga que nos presentan en el video de David, pero no quiere decir que en el primer problema que nos presentaron de la fábrica no se hayan relacionado estos aspectos, por ejemplo un primer concepto que asociamos con el problema original fue con el cambio que se presentaba de un valor a otro, tal y como lo mencionaba David, pues nos pedían en cierta forma hacer ciertas variaciones entre la cantidad de productos (motores, hélices) para encontrar el mayor costo de producción”. [Sesión 28, G2, informe síntesis p.6].

En este escrito Edna y Adriana están intentando describir un significado general 
del concepto de variación comparando los dos problemas, tratando de adecuar la noción de cambio que presenta David con la que ponen en juego en el problema de la fábrica. Sin embargo, cuando desean precisar el significado de la dependencia entre los cambios hacen una alusión explícita a la dependencia funcional. Asumen que el problema de optimización responde, en cierta forma, a la cuantificación de la relación entre las variaciones de una variable que genera variaciones en la otra.

Esta descripción pone de manifiesto que han construido un significado de la variación identificando variables, parámetros, cuantificadores, así como la relación funcional que puede ser aplicada a las dos situaciones. A su vez, en la intervención de Gustavo integrante de G2, se explicita un significado de covariación asociado a su problema:

“... si por ejemplo nuestro caso el costo de producción era la razón de cambio que habia entre la variación de las hélices o la razón de cambio de las hélices y de los motores, entonces aquí, sino que en este caso no eran tan dependientes, ¿si me entienden? Sólo la primera que actuara era la que importaba para que las demás actuaran ¿sí? Entonces eso en lo que tiene que ver con esa pregunta..." [Sesión 28, G2, socialización en gran grupo].

El significado de covariación que expone Gustavo da cuenta de la influencia de las cantidades de cada producto a fabricar en relación con la proporción que cada unidad de cada producto aportaba al costo total. En la respuesta a la pregunta de Juana, integrante de G3, observamos que son conscientes de que la derivada como modelo matemático que mide la razón de cambio en una variable no fue utilizada en la solución de su problema (el de la fábrica), ya que éste involucra otro modelo matemático: la optimización en el contexto de la programación lineal.

Juana: es que yo quería saber, dos cosas, la primera es [...] ¿Cuál es la idea que tienen ahora de variación? y, como el problema de ustedes es distinto [...] ¿Qué construcción hicieron con ese problema? [...] ¿Qué objeto matemático construyeron ustedes con la primera situación [el problema de la fábrica] y ¿qué [...] noción de variación tienen hoy?

Edna: pues digamos, lo que nosotros decíamos era que en el primer ciclo nosotros pensábamos que ahi se trabajaba con derivada pero terminamos metiéndonos como por Cantor, ese fue nuestro objeto matemático.

Adriana: o sea, la solución del primer problema, del problema inicial, el que teníamos nosotros, también tenía que ver mucho con lo que era programación lineal y optimización convexa que fue lo que vimos en la primera exposición que hicimos... [Sesión 28, G2, exposición en gran grupo].

En lo anterior se aprecia una manifestación de una construcción del significado de variación como la cuantificación del cambio. Esta manera de entender la variación integra los significados que emergen de la actividad desplegada para resolver cada uno de los dos problemas. Es decir, como casos particulares de la manera de cuantificar el cambio en los contextos razón de cambio y programación lineal.

\subsection{Construcción de una comunidad de aprendices}

La emergencia de una comunidad de aprendices se constituyó en este experimento de enseñanza como un mecanismo importante en el proceso de construir el significado de la variación matemática desde la perspectiva de la enseñanza de las matemáticas. Para dar cuenta de esta influencia, identificamos tres aspectos que hacen emerger una 
comunidad de práctica: construir dominios comunes de interés, compartir información y desarrollar un repertorio de recursos compartidos.

Construyendo dominios comunes de interés sobre los elementos matemáticos que constituyen el concepto de variación

Durante el experimento de enseñanza los estudiantes para profesor intentaron definir en tres ámbitos un dominio común de interés a través de los cuales identificar y compartir los significados generados: la determinación de parámetros, el proceso de simbolización para representar la variación y el uso de las restricciones.

En primer lugar, la determinación de parámetros evidenció cómo los estudiantes focalizaban la atención y determinaban la importancia de llegar a compartir lo que cada uno estaba interpretando. Así, en la sesión 10, G6 expone su proceso de solución del problema de la viga, describiendo las características de las secciones rectangulares indicando su confusión al obtener dos ecuaciones que solucionan el problema pero con resultados diferentes. En ese momento, sus compañeros intervienen para llamar la atención sobre lo que implica que la sección rectangular esté inscrita. Es decir, las características de las variables y su relación con la restricción se vuelven objeto explícito de discusión.

David: Ustedes están reemplazando en esta fórmula de resistencia igual a $R=k a h^{2}$

G6: $\quad S i$

David: Y eso qué tiene que ver con la fórmula de $k a \varphi^{2}-a^{2}$ ¿Da lo mismo si ustedes reemplazan los datos en esa fórmula y en la que ustedes usaron? Y ¿obtienen los mismos datos?...porque creo que no

G6: $\quad$ No

David: entonces la pregunta sería ¿cuál tendrían en cuenta? Esa que obtuvieron a partir de la relación con el diámetro $\left[k a \varphi^{2}-a^{2}\right]$ o esa $\left[R=k a h^{2}\right][\ldots]$ porque en esta $\left[R=k a h^{2}\right]$, de alguna forma no están teniendo en cuenta la relación que tienen las dimensiones con el diámetro, o sea allá $\left[k a \varphi^{2}-a^{2}\right]$ si la consideran, pero acá no $\left[R=k a h^{2}\right]$ y allá,..., están perdiendo la relación que ya habian ganado.

Un segundo momento importante en el proceso de construir dominios compartidos de interés relevante para dotar de significado a la variación matemática estuvo relacionado con la forma en la que llegaron a compartir maneras de simbolizar la covariación. Por ejemplo, en la sesión 15 David expone el proceso de solución al problema de la viga e identifica como relevante la importancia del parámetro, de las restricciones y de las relaciones entre variables, para dar cuenta de la covariación:

La variación que hay entre la variación del ancho [representado en el eje $\mathrm{x}$ de la figura 5] con la variación de la resistencia [representado en el eje y de la figura 5], que tendríamos algo como esto [señalando la representación en el tablero]. La variación que presenta la resistencia con respecto a la variación que está presentando el ancho aqui en cada parte de la parábola [señala la curva]. Pero realmente lo que estamos haciendo es esto: cuando estamos hablando de que estamos analizando la relación que hay entre la variación de la resistencia y la variación de "a" esa relación nos da como estas rectas que se forman.

No nos está dando estos puntos que es como una aproximación; es una aproximación de pronto a la parábola pero en cierta medida de pronto no es exactamente la parábola [señala la curva]. Si bien nos lleva al manejo de la relación entre el ancho y la resistencia vemos que pasa esto. [Sesión 15, David, exposición en gran grupo]. 


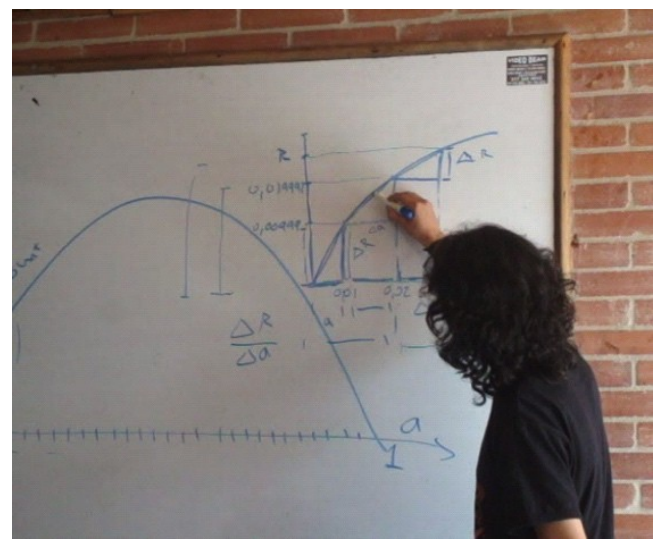

Figura 5. David explicando una aproximación a la medida de la variación

Esta intervención es un intento de David de ayudar a los otros a entender cómo dar cuenta de la covariación haciendo explícitas las relaciones entre los incrementos en la variable $x$ y su relación con los incrementos en la variable $y$, considerados como los elementos que la constituyen. En las interacciones siguientes los otros estudiantes comparten con David sus apreciaciones sobre los incrementos, sus relaciones y la manera de simbolizarlos.

David: $\quad$ por eso, pero acá es que "a más algo"... vamos a irnos al caso particular ¿listo? Tenemos "a" ¿cierto? Eso es "a". Pero estamos hablando del caso particular, sabemos implícitamente que es 0.01; el siguiente " $a$ " es éste +0.01 ¿cierto? Para el caso particular. Entonces, ¿qué es lo que estamos agregando al siguiente? Pues como el anterior, pues la unidad de medida que es lo que yo dije.

Juana: ¿Lo estableció en el "zoom"?

David: $\quad$ el siguiente es "a más 0.01" y el siguiente va a ser esto $+0.01 \ldots$ va a ser así... el siguiente va a ser esto más 0.01 .

Leidy: pero si la idea es de pronto es generalizar, ¿cómo va a trabajar para cualquier caso y eso que estás haciendo te va a servir para cualquier caso?, lo que puedes hacer es en vez de poner " $a+1$ ", pues no es correcto, podrías poner un $a_{1} o$ " $a+b$ " donde " $b$ " sería la constante dentro de la unidad de medida, o " $b$ "; " $b$ " solito que sería el siguiente número.

David: pues sería algo como esto...

Leidy: No, $a+a_{1}$ no " $a+b$ " $y$ " $b$ " sería la constante de la unidad de medida que es lo que estás diciendo.

David: $\quad$ Ese es un ancho... y ese ancho es un $a_{i}$, el siguiente ancho pongámoslo en términos... sería " $a$ ", un nuevo " $a$ " ...

Juana: $\quad$ No, entonces sería $b-a_{1}$ [Sesión 15, David, exposición en gran grupo]

Gran grupo: Póngale h....

David: $\quad$ Vamos a hacer lo siguiente... a ver si ya de pronto unimos ideas... estábamos viendo que acá le sumo algo, es un "algo" ¿listo? Ese algo lo vamos a poner en términos de una letra: de " $h$ ". ¿Listo? para cualquier caso. Acá tenemos " $a$ " que puede ser cualquier medida y tenemos eso de acuerdo a esa idea. ¿Estamos entendiendo lo que pasa? [Sesión 15, David, exposición en gran grupo].

Las distintas intervenciones de los estudiantes sobre la manera de expresar las 
variaciones del ancho indican que el intento de David para ayudar a sus compañeros a comprender la covariación y su simbolización ha sido exitoso y que David ha incorporado en su discurso los significados expresados por sus compañeros en la discusión en el gran grupo. Este proceso de llegar a usar unos símbolos comunes se concreta con la introducción de $\mathrm{h}$, como una manera de expresar el incremento constante de la variación del ancho y dar cuenta de la medida de la covariación.

Finalmente, otro dominio de interés común fue el conocimiento del uso de las restricciones para expresar la función a utilizar en términos de una sola variable, que se evidencia por las interacciones de los estudiantes, descritas en la viñeta 1, en un intento por ponerse de acuerdo. En sus discusiones los estudiantes ponen de manifiesto intentos por comprender la manera en la que los otros usan la restricción (relación pitagórica) para ir construyendo un significado compartido sobre la importancia del uso de la misma para establecer la relación funcional. La manera en la que los estudiantes consideran la restricción de las variables y su importancia para establecer la relación funcional y la covariación fue un elemento clave del proceso de construcción del significado común de los elementos constitutivos de la variación matemática.

\section{Compartiendo información}

Los momentos en que los estudiantes compartían información permitieron que se favoreciera la posibilidad de aprender unos de otros. Esta característica se manifestó en las sesiones en gran grupo en donde las diferentes intervenciones posibilitaban la construcción de un significado compartido. Por ejemplo en la sesión 10, G6 había presentado su solución al problema y al finalizar la exposición algunos compañeros discutieron sobre el procedimiento usado identificando dificultades en el mismo:

Lady: lo que pasa es que si ellos [dirigiéndose al gran grupo] dicen que la mayor altura que tienen en el círculo es igual al diámetro, pero no puede ser igual al diámetro, lo que pasa es que a ellos lo que les falta es condicionar un poco las medidas y hacer las alteraciones más pequeñas, porque por ejemplo cuando nosotros hicimos el nuestro hacíamos las alteraciones, digamos de 0.5, pero a medida que hacíamos las alteraciones cada vez más pequeñas nos dábamos cuenta que la resistencia iba aumentando, entonces las alteraciones o ustedes las están haciendo muy grandes o están tomando la altura, o sea, la altura como muy alta y si se están dando cuenta, digamos en este sentido, que la altura va a caber exactamente dentro de la circunferencia, hay que tener en cuenta que no se puede salir de la circunferencia, ni que puede ser igual al diámetro porque sería una linea.

Julián: al principio de la exposición ustedes dijeron que las condiciones eran que $h$ no puede ser igual a $\varphi$ [diámetro], $h$ tenía que ser menor que $\varphi$ y que h tenía que ser mayor que a.

Lady: y por ejemplo en lo que nosotros quedamos fue que $h$ si tiene que ser mayor que a, pero que no podía ser cualquier número porque o si no la resistencia no iba a ser la mayor, tienen que tener una relación, tienen que tener una condición para establecer las medidas. [Sesión 10, G6, exposición en gran grupo].

Este tipo de interacciones dadas a lo largo del experimento de enseñanza pone de manifiesto la manera en la que los estudiantes comparten información y la intención con la que la comparten. Ponen en juego la experiencia ganada en el proceso de resolución dentro del pequeño grupo y en las exposiciones en gran grupo. En algunos 
momentos atienden a las relaciones matemáticas que constituyen el problema y su solución, en otros momentos tematizan las posibles cuestiones problemáticas emergentes en los procesos de resolución externalizados por los otros. En el primer caso la atención se dirige a la solución del problema matemático, en el segundo caso la atención se dirige a generalizar su toma de conciencia de las cuestiones exhibidas y arrojar luces para entenderlas y superarlas.

\section{Desarrollo de un repertorio compartido de recursos}

El desarrollo de un repertorio compartido de recursos se reflejó en los usos de la simbología matemática y en el discurso matemático generado. Esto fue motivado por el diseño del curso que incluía interacciones en pequeños grupos y en gran grupo dando cabida a la participación de los estudiantes asumiendo diferentes roles y responsabilidades. Por ejemplo, en la sesión 15, David está expresando simbólicamente los incrementos en los ejes de coordenadas, simbolizándolos como $a$ y $a+1, f(a)$ y $f(a+1)$ respectivamente, los otros estudiantes le llaman la atención sobre la manera en que lo está haciendo debido a que toma el valor del incremento como una constante y debe ser una variable simbolizada.

Estudiante 1: No sé si ese +1 no es de la unidad de medida que se está trabajando...

David: $\quad$ sí, tiene razón. Acá sería eso...

Estudiante 1: Eso

David: ¿si?

Gustavo: ¿no debería ser desde un $a_{i}$ hasta un $a_{j}$ ?

David: $\quad$ Entonces sería asi: (Figura 6)

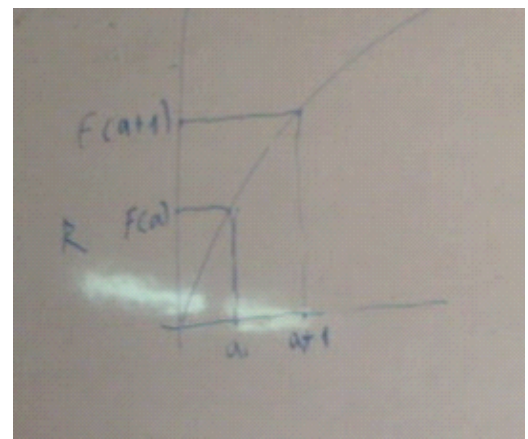

Figura 6. Representación usada por David durante el proceso de simbolización Gran grupo: No.

Gustavo: El "a" está bien pero entonces el siguiente sería "a" más un "a" que usted no conoce y ese "a" también varía por la continuidad, la misma continuidad que usted estaba diciendo.

Gran grupo: [...Murmullos]

David: $\quad$ es la misma interpretación $[\ldots]$

David: $\quad$ Vamos a hacer lo siguiente... a ver si ya de pronto unimos ideas... estábamos viendo que acá le sumo algo, es un "algo" ¿listo? Ese algo lo vamos a poner en términos de una letra: de " $h$ ". ¿Listo? Para cualquier caso. [Sesión 15, David, exposición en gran grupo]. 
La aceptación por todos de que h es un símbolo general que es utilizado para definir la covariación como razón de cambio y la construcción de una simbología común y más eficaz les posibilita compartir un recurso representacional que unifica maneras de expresarse por todos entendida.

\section{Discusión y conclusiones}

Dos ideas emergen de los resultados aportados en relación a la construcción del significado de variación matemática en estudiantes para profesor cuando participan en un entorno de aprendizaje diseñado desde una perspectiva que enfatiza la dimensión sociocultural del aprendizaje. En primer lugar, las características del proceso de construcción del significado variación matemática como emergente de la práctica de resolución de problemas. En segundo, la identificación de factores que influyeron en la constitución de una comunidad de aprendizaje.

\subsection{Características del proceso de construcción del significado}

En relación al proceso de construcción del significado de la variación matemática nuestros resultados indican que este proceso de construcción se apoyó en la consideración de los diversos contextos de variación matemática. En este sentido, nuestros resultados pueden ser interpretados como intentos exitosos de comprensión por parte de los estudiantes para profesor de integrar los significados de la variación en dos contextos: 1) la modelada por la derivada como razón de cambio que cuantifica la covariación entre magnitudes, y 2) la modelada por la programación lineal que cuantifica tanto la covariación entre magnitudes discretas, como la proporción del cambio de la función objetivo ocasionado por el cambio por unidad de cambio en las magnitudes discretas. La comprensión de la primera clase de variación matemática se apoyó en el hallazgo de la relación entre el conjunto de condiciones relativas a la forma de la viga y la forma de calcular la resistencia y su conversión en una relación de tipo instrumental. Para ello los estudiantes para profesor tuvieron en cuenta la relación entre: un conjunto de parámetros, las variables que intervienen, las restricciones y cuantificadores junto con sus dominios de actuación y de validez, y la función objetivo. Esta relación permitió a los estudiantes para profesor generar una representación algebraica de la función objetivo como una función en una sola variable real y de valor real. Dicha comprensión apoyó los intentos de asignar significado a la expresión "pequeñas alteraciones" y de establecer la relación con el hallazgo de la solución óptima, junto con la derivada como razón de cambio en tanto manera de cuantificación de la covariación.

Por otra parte, la comprensión de la variación matemática en el caso de las magnitudes discretas, pasó por el hallazgo de la relación entre el conjunto de condiciones relativas a la capacidad de la fábrica de asumir un costo total de producción y los costos de producción por motor y por hélice, teniendo en cuenta la forma de calcular el costo total de producción. En este caso también se generó la relación entre: un conjunto de parámetros, las variables, las restricciones y cuantificadores, junto con sus dominios de actuación y de validez, y la función objetivo. Sin embargo, en este caso también se tuvo en cuenta la determinación de la influencia de las cantidades de cada producto a fabricar en relación con la proporción que cada unidad de cada producto aportaba al costo total. En este caso, el uso de las tablas como heurísticas para generar y organizar información numérica, utilizadas 
durante el proceso de resolución, permitió la generación de procesos de negociación de las relaciones numéricas y de las formas de simbolización para describir estas relaciones.

\subsection{Constitución de una comunidad de aprendices}

En relación a la segunda idea, la constitución de una comunidad de aprendices, la actuación de G2 que resolvió inicialmente el problema de la Fábrica y las discusiones colectivas a partir de la presentación de David permitió evidenciar de qué manera los estudiantes identificaron objetivos comunes constituyendo espacios de intercambio y desarrollando recursos compartido como aspectos relevantes de una comunidad de aprendices.

En el primer ciclo de resolución G2 mostró un dominio del significado de la variación matemática que permitió generar la conciencia de que lo que compartían con los otros grupos definía un dominio común. Este objetivo común era la variación matemática como concepto matemático emergente de la resolución de las dos clases de problemas. Los estudiantes llegaron a ser conscientes de que la práctica en los grupos y las técnicas involucradas en los procesos de resolución eran diferentes (en particular entre los de G2 comparados con los de los demás). Este hecho creó la necesidad de construir un discurso común para hablar de variación como un concepto general que generó procesos de negociación de los significados de la variación matemática y sus particularidades continua y/o discreta. En particular, cuando G2 decide abandonar la generalización de la clase de variación modelada por la programación lineal y adecuarse a la clase modelada por la derivada se adapta al discurso matemático predominante en el aula con el propósito de lograr una identidad (Wenger, 2001) adaptando una práctica local (la variación en contexto discreto) a una práctica global (la variación matemática) para pasarse con legitimidad a la otra práctica local (la variación en contexto continuo). Nosotros podemos interpretar que la legitimidad para ir adaptando el discurso procede de una valoración social estimulada a través de los textos de cálculo y de los lineamientos curriculares que proponen el significado de variación matemática casi exclusivamente ligado al modelo diferencial y un andamiaje social asimétrico (Speer y Wagne, 2009). Este contexto asimétrico conllevó que G2 tuviera que analizar la resolución del otro problema para participar en la discusión del video de David en el que se presentaba la resolución del problema en contexto continuo. Sin embargo, podemos asumir que esta asimetría impulsó a los estudiantes de G2 a generalizar la clase de variación modelada por la programación lineal reconociendo las diferencias y semejanzas con lo realizado por los demás grupos.

Debido a ello, creemos que en este tipo de situaciones el diseño de las clases debería contemplar una mayor simetría para nivelar el número de grupos abordando los dos tipos de problemas. Este hecho podría favorecer la constitución de la complementariedad de los significados apoyados en el proceso de negociación derivados de la necesidad de compartir recursos (discursos y formas de simbolizar) y dominios de interés compartido.

Finalmente, la hipótesis inicial en el diseño del experimento de enseñanza que considera que la formación matemática y didáctica de los futuros profesores puede darse de manera complementaria, fue apoyada por el diseño realizado. En particular, el primer ciclo tuvo como propósito que los estudiantes para profesor participaran en un entorno de aprendizaje focalizado en la práctica de resolver problemas de variación 
matemática. El objetivo era ayudar a generar un proceso de significación del concepto de variación matemática en actividades que involucraban dos clases de variación. Esto llevó a los grupos de estudiantes a tomar a su cargo sesiones plenarias en las que compartieron con el resto de compañeros reflexiones acerca de sus procesos de solución: heurísticas, atascamientos, avances, errores y correcciones, redireccionamientos del proceso, usos de instrumentos físicos y conceptuales, argumentaciones, etc. Este estilo de participación condujo a los estudiantes a asumir el rol de profesor manifestando la intencionalidad de promover que otros aprendan y tomando conciencia de esta forma de aprendizaje. Estos aspectos consideramos que apoyaron el sentido de ser profesor en los participantes en este experimento. Aunque nuevas investigaciones deberían centrarse en estos últimos aspectos, creemos que los resultados aportados por nuestra investigación señalan el efecto sobre el aprendizaje de los estudiantes para profesor del hecho de apoyar las reflexiones didácticas sobre la gestión de la actividad matemática en las situaciones de resolución de problemas en las que han participado.

\section{Agradecimientos.}

Los análisis reportados en este artículo son producto de la investigación "El uso de problemas matemáticos como instrumentos de aprendizaje en la formación de profesores" financiado por Colciencias y la Universidad Distrital Francisco José de Caldas. (Cod. 1130-452-21063).

Agradecemos a los estudiantes del Proyecto Curricular de Licenciatura en Educación Básica con énfasis en Matemáticas (LEBEM), que cursaron Didáctica de la Variación en el segundo semestre de 2009 por permitirnos desarrollar con ellos la propuesta constitutiva del Experimento de Enseñanza.

Un reconocimiento especial al apoyo y asesoría del Doctor Salvador Llinares Ciscar, Universidad de Alicante-España.

\section{Referencias bibliográficas}

Artigue, M. (1991). Analysis. In D. Tall (Ed.). Advanced Mathematical Thinking (pp. 167198). Dordrecht, The Netherlands: Kluwer.

Artigue, M., Batanero, C., \& Kent, P. (2007). Mathematics thinking and learning at postsecondary level. In F. K. Lester (Ed.). Second Handbook of Research on Mathematics Teaching and Learning (pp.1011-1050). Reston: National Council of Teachers of Mathematics (NCTM).

Biza, I., Nardi, E., \& Zachariades, T. (2007). Using tasks to explore teacher knowledge in situation-specific contexts. Journal of Mathematics Teacher Education, 10(4\&6), 301309.

Bohórquez, L., Bonilla, M., \& Romero, J. (2009). Los problemas matemáticos en la construcción de la idea de variación y la caracterización de problema de variación. En Memorias Décimo Encuentro Colombiano de Matemática Educativa, ASOCOLME (pp. 145-160). Bogotá: GAIA.

Cantoral, R., \& Farfán, R. (1998). Pensamiento y lenguaje variacional en la introducción al análisis. Epsilon, 42(14), 353-369.

Cantoral, R., Molina, J., \& Sánchez, M. (2005). Socioepistemología de la predicción. México: Cinvestav IPN. 
Cobb, P., Confrey, J., diSessa, A., \& Lehrer, R. (2003). Design Experiments in Educational Research. Educational Researcher, 32(1), 9-13.

Conner, A., Wilson, P. S., \& Kim, H. J. (2011). Building of mathematical events in the classroom. ZDM Mathematics Education, 43, 979-992.

Design-Based Researcher Collective (2003). Design- Based Research: An Emerging paradigm for Educational Inquiry. Educational Researcher, 32(1), 5-8.

Dreyfus, T. (1990). Advanced mathematical thinking. In P. Nesher and J. Kilpatrick (Eds.), Mathematics and Cognition: A Research Synthesis by the International Group for the Psychology of Mathematics Education (pp. 113-134). ICMI Study Series. Cambridge: Cambridge University Press.

Fernandes, D., Lester, F. Borralho, A., \& Vale, I. (Coords.). (1997). Resoluçao de problemas na formaçao inicial de professores de matemática. Múltiplos Contextos e Perspectivas. Aveiro: GIRP.

García, M., Llinares, S. \& Sánchez-Matamoros, G. (2011). Characterizing thematized derivative schema by the underlying emergent structures. International Journal of Science and Mathematics Education, 9(5):1023-1045.

Gavilán, J., García, M., \& Llinares, S. (2007). Una perspectiva para el análisis de la práctica del profesor de matemáticas. Implicaciones metodológicas. Enseñanza de las Ciencias, 25(2), 157-170.

Gravemeijer, K. (2004). Local Instruction Theories as Means of Support for Teachers in Reform Mathematics Education. Mathematical Thinking and Learning, 6(2), 105-128.

Lave, J., \& Wenger, E. (1991). Situated learning. Legitimate peripherical participation. Cambridge: Cambridge University Press.

LEBEM. (1998). Documento base del Proyecto Curricular de Licenciatura en Educación Básica con Énfasis en Matemáticas. Bogotá: Universidad Distrital.

Sánchez-Matamoros, G., García, M. \& Llinares, S. (2006). El desarrollo del esquema de derivada. Enseñanza de las Ciencias, 24, (1), 85-98.

Sánchez-Matamoros, G., Fernández, C. \& Llinares, S. (2014) Developing pre-service teachers' noticing of students' understanding of the derivative concept. International Journal of Science and Mathematics Education, DOI: 10.1007/s10763-014-9544-y.

Santos-Trigo, M., \& Camacho-Machín, M. (2009). Towards the Construction of a Framework to Deal with Routine Problems to Foster Mathematical Inquiry. PRIMUS: Problems, Resources and Issues in Mathematics Undergraduate Studies, 19(3), 260-279.

Selden, A., \& Selden, J. (2001). Tertiary Mathematics Education Research and its Future. In D. Holton (Ed.). The Teaching and Learning of Mathematics at University Level (pp. 237254). Dordrecht, The Netherlands: Kluwer Academic Publishers.

Silver, E. A., Clark, L. M., Ghousseini, H. N., Charalambous, C. Y., \& Sealy, J. T. (2007). Where is the mathematics? Examining teachers' mathematical learning opportunities in practice-based professional learning tasks. Journal of Mathematics Teacher Education, 10, 261-277.

Simon, M. (2000). Research on the Development of Mathematics Teachers: The teacher Development Experiment. In A. Kelly \& R. Lesh (Eds.), Handbook of Research Design in Mathematics and Science Education (pp. 335-359). Mahwah, NJ: Lawrence Erlbaum Associates.

Sowder, J. (2007). The Mathematical Education and Development of Teachers. In F.K. Lester (Jr.) (Ed.). Second Handbook of Research on Mathematics Teaching and Learning (pp.157-224). IAP-NCTM: Charlotte, NC. 
Steffe, L., Thompson, P., \& von Glasersfeld, E. (2000). Teaching experiment methodology: Underlying principles and essential elements. In A. Kelly \& R. Lesh (Eds.). Handbook of Research Design in Mathematics and Science Education (pp. 267-306). Mahwah, NJ: Lawrence Erlbaum Associates.

Speer, N., \& Wagne, J. (2009). Knowledge Needed by a Teacher to Provide Analytic Scaffolding During Undergraduate Mathematics Classroom Discussions. Journal for Research in Mathematics Education, 40(5), 530-562.

Wenger, E. (2001). Comunidades de práctica. Aprendizaje, significado e identidad. Buenos Aires: Paidós.

\section{Referencias a los autores}

Martha Bonilla Estévez, Universidad Distrital Francisco José de Caldas (Colombia), marthaedumat@udistrital.edu.co

Jaime Romero Cruz, Universidad Distrital Francisco José de Caldas (Colombia), jaimeedumat@udistrital.edu.co

Deissy Narváez Ortiz, Universidad Distrital Francisco José de Caldas (Colombia), dmnarvaez@gmail.com

Angel Bohórquez Arenas, Universidad Distrital Francisco José de Caldas (Colombia), angel.bohorquez@gmail.com 


\title{
Characteristics of the building process of mathematic variation concept meaning in prospective teachers of mathematics
}

\author{
Martha Bonilla Estévez, Universidad Distrital Francisco José de Caldas (Colombia) \\ Jaime Romero Cruz, Universidad Distrital Francisco José de Caldas (Colombia) \\ Deissy Narváez Ortiz, Universidad Distrital Francisco José de Caldas (Colombia) \\ Angel Bohórquez Arenas, Universidad Distrital Francisco José de Caldas (Colombia)
}

This article presents some of the results from a teaching experiment developed during the Didactics of Variation class of the undergraduate program in Elementary and Middle School Teaching with Emphasis in Mathematics. The purpose of the experiment was to inquire about the building process of the meaning of mathematical variation in a learning environment that combined problem solving and participating in a community of learners.

In the experiment, twenty-four prospective teachers participated. It took twenty-eight twohour sessions grouped in three cycles. Class methodology was based on the perspective of problem solving and an organization that combined individual work, work in small groups and presentations with the whole group.

The problems posed were thought so that students would generate an idea of variation that involves recognizing variables, parameters, quantifiers, functional relations and covariation relations, and that can be modeled in optimization contexts. In particular, the derived as a measure of velocity change in a continuous context and linear programing as a modeling context of covariation with discrete magnitudes.

Data from sixteen students organized in four groups, three of which solve the beam problem and only one the fabric problem, was collected. This fact generated a context of meaning interchange throughout the collective discussions of the whole group, determining the meaning of mathematical variation encompassing discrete and continues contexts.

In relation to the building process of the meaning of mathematical variation our results, described through two vignettes, can be interpreted as successful attempts, from the prospective teachers, to integrate in a comprehensible manner the meanings of the variation in the two chosen contexts: 1) the variation modeled by the derived as the change rate that quantifies the covariation between magnitudes, and 2) the variation modeled by linear programming that quantifies both the covariation between discrete magnitudes and the proportion of the change of the target function caused by the change per change unit in the discrete magnitudes.

Related to the conformation of the learners community, we show the process trough which the students identified that the common goal was mathematical variation as an emerging concept from the solving of both kinds of problems. Also, since they became aware that the practice inside the groups and the techniques involved in the solving processes were different, the necessity of building a common discourse to talk about variation as a general concept appeared, thus evidencing negotiation processes of the meaning of mathematical variation and particular characteristics of the continuous and discrete variation.

The kind of participation designed for the whole group presentations drove the students to assume the teacher's role, manifesting the intention to promote learning in others and becoming aware of this way of learning. This last, also supports the building of the teacher's identity. 